\title{
Case Report: The Role of Spatial Repellant Devices to Prevent Malaria in Low-Income Countries
}

\author{
Giovanni Cucchiaro, ${ }^{1 *}$ Jamie Van Leeuwen, ${ }^{2}$ and Yvette Goodridge ${ }^{3}$ \\ ${ }^{1}$ Department of Anesthesiology and Critical Care Medicine, Johns Hopkins University, Johns Hopkins All Children's Hospital, St. Petersburg, \\ Florida; ' Global Livingston Institute, Denver, Colorado; ${ }^{3}$ Department of Anesthesiology and Critical Care Medicine, Children's Hospital Los \\ Angeles, Los Angeles, California
}

\begin{abstract}
Malaria is the leading cause of morbidity and mortality in Uganda. The role of spatial repellent devices in preventing malaria is controversial. The goal of this study was to evaluate the populations' acceptability of a newly designed insecticide diffuser. We distributed to three families living in southern Uganda a device commercially available, the VAPE ${ }^{\circledR}$ portable set. This spatial repellent device offers several advantages compared with other traditional products. It is powered by lithium batteries that guarantee 20 days of uninterrupted delivery of insecticide; it contains two insecticides: empenthrin and transfluthrin; and it is simple to use, one switch to turn it "on" and/or "off." It is odorless, and it can be placed anywhere in the living/sleeping area. People can also carry it outside the house. We planned to evaluate people's compliance with its usage, its reliability, and its overall costs. We conducted a 5-month survey. We distributed the devices to three households, one device per bedroom. Ten males and 11 females, with a mean age of $26 \pm 16$ (range 10-51) years, lived in these houses. The compliance with the use of the device and its acceptability were high. No side effects were reported. No individual contracted malaria during the 5-month period. The major obstacle we found was the timely delivery of the devices to the evaluation area and initial compliance with the instructions on how to use the device. Larger randomized studies are needed to clarify whether there is a role for this type of spatial repellent devices in the global efforts to prevent malaria.
\end{abstract}

\section{INTRODUCTION}

There have been significant gains in the fight against malaria over the past 15 years. Since 2000, 12 countries have eliminated malaria; the WHO certified four countries as malaria free between 2007 and 2013, and an additional eight countries were moved into the WHO's prevention-of-reintroduction phase after sustaining at least 3 years of zero local malaria transmission. ${ }^{1}$ The fight against malaria requires a multipronged approach, and the use of long-lasting insecticidal nets (LLINs) and indoor residual spray of insecticides has contributed to its success. However, there still are countries where malaria is the leading cause of morbidity and mortality. In Uganda, malaria accounts for 30-50\% of outpatient visits at health facilities, $15-20 \%$ of all hospital admissions, and up to 20\% hospital deaths. ${ }^{2,3}$ The Uganda Ministry of Health recorded 1.4 million cases of malaria in June 2019, a 40\% surge. ${ }^{4}$ The high LLIN's attrition with subsequent costs and distribution problems have been identified as one of the most common causes of persistent malarial infections in the country. ${ }^{5}$

We conducted a health-related project in two villages in southern Uganda using a new type of insecticide diffuser, the VAPE $^{\circledR}$ portable set (VPS) (Henkel, Germany) (Figure 1). The device is sold in supermarkets in Europe, and it can be bought online. It is powered by lithium batteries that guarantee 20 days of uninterrupted delivery of insecticide; it is filled with micro-granules containing transfluthrin ${ }^{6}$ and empenthrin ${ }^{7}$; and it is simple to use, one switch will turn it on and/or off. It is odorless, and it can be placed anywhere in the house. People can also carry it outside the house where it provides protection for people standing within a 10 -feet radius.

\footnotetext{
* Address correspondence to Giovanni Cucchiaro, Department of Anesthesiology and Critical Care Medicine., Johns Hopkins All Children's Hospital, 601 5th St. South, St. Petersburg, FL 33701. E-mail: gcucchi1@jhmi.edu
}

The goal of this project was to collect some baseline data on compliance, reliability, and acceptance of this device in a lowincome rural community.

\section{MATERIALS AND METHODS}

We conducted the evaluation in two villages located in the Kabale district (south Uganda) (Figure 2). Ten VPSs were bought from a supermarket in Europe. After discussions with several families, we randomly chose three families that agreed to participate in this evaluation. Twenty-one individuals lived in these families. The Global Livingston Institute (GLI), a Ugandan-run community development organization based in the region that has served the local population for a decade, provided organizational and personnel support. ${ }^{8}$ No incentives were offered to families, and the participation was strictly voluntary. We described the functions of the VPS and gave instructions on its use. VAPE portable set is childproof to prevent access to the micro-granules, and access could only occur if the device is intentionally cracked. We instructed to turn it on at 18:00 PM and off at 6:00 AM. Based on this schedule, the devices would offer a 40-day coverage before the micro-granules and/or the batteries needed to be replaced. The GLI team initially assisted with changing batteries and micro-granules, and the families, if comfortable, would do it independently after the second recharge. We collected demographic and health data. We recommended that families place one VPS in each room, to wear the device when going outside to cook or for a walk. The principal investigator and GLI staff visited families daily for the first 72 hours to verify the correct utilization of the VPS. The GLI personnel then visited the families every 2 weeks to verify that the devices were still operational and changed the batteries and/ or the micro-granules as needed while looking for signs of malaria or side effects from the devices (asthma-like respiratory symptoms). We recommended going to the local health clinic for any symptom suggesting malaria to rule out malaria using a rapid diagnostic test (RDT) kit (Corestart ${ }^{\text {TM }}$ 


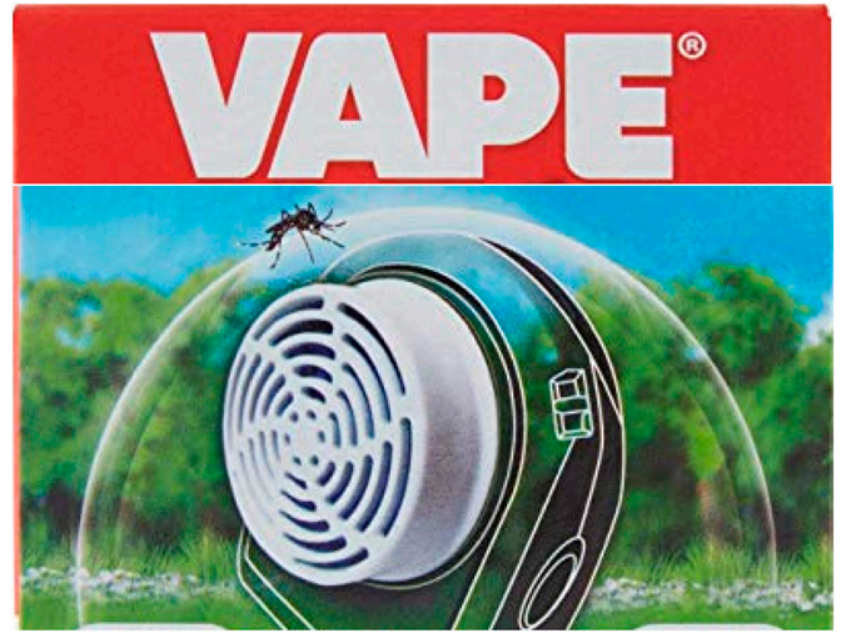

FIGURE 1. VAPE portable set. This figure appears in color at www.ajtmh.org.

Malaria Screen, ACCESSBIO, Addis Ababa, Ethiopia). Every family had at least one cell phone, and they were instructed to contact the GLI staff for questions on health problems. Families were encouraged to continue with their customary habits with respect to actions that could prevent malaria.

\section{RESULTS}

Twenty-one subjects from three families were evaluated (Table 1). Twelve (60\%) had at least one episode of malaria in the prior 12 months, which was confirmed by a review of the local health clinic records. We distributed 10 devices, one device per bedroom. There was no evidence that any of the families used bed nets, and $100 \%$ of the subjects confirmed that they used them very sporadically, if at all.

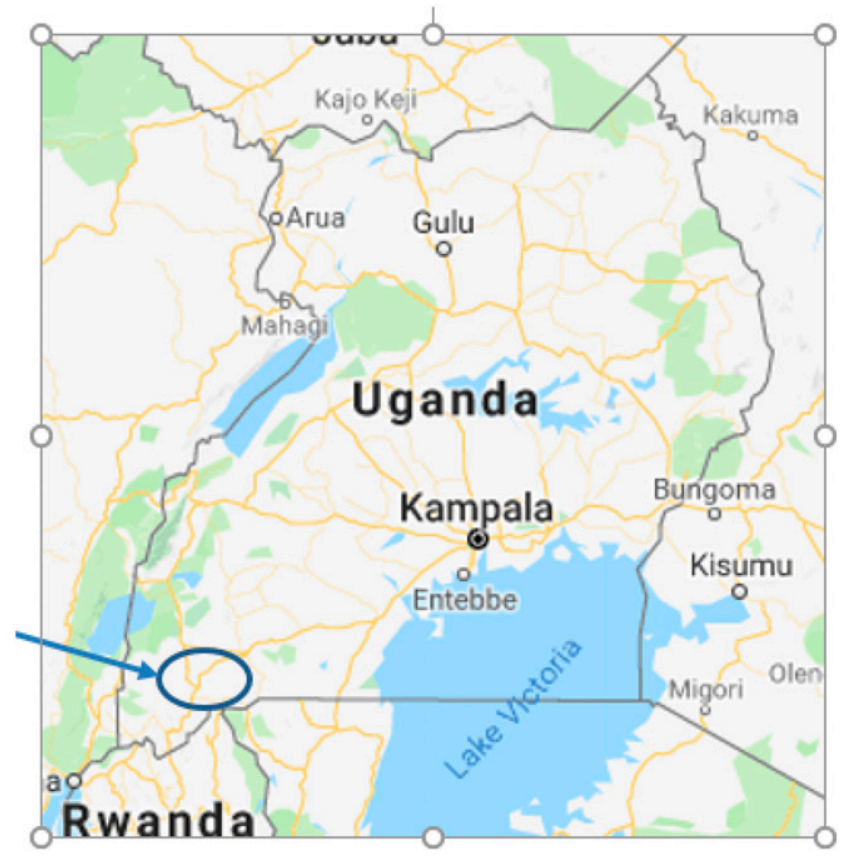

FlgURE 2. Map showing Kabale district in Uganda. This figure appears in color at www.ajtmh.org.
TABLE 1

Population demographic data

\begin{tabular}{lccc}
\hline & Family 1 & Family 2 & Family 3 \\
\hline Number of members & 6 & 10 & 5 \\
Age (years) & $30 \pm 18$ & $25 \pm 14$ & $22 \pm 15$ \\
Gender (m/f) & $4 / 2$ & $6 / 4$ & $0 / 5$ \\
Number of bedrooms & 3 & 4 & 3 \\
Number of people contracted malaria & $4 / 5$ & $6 / 10$ & $3 / 5$ \\
$\quad$ previous 12 months (m/f) & & & \\
$\quad \begin{array}{l}\text { Number of episodes of malaria } \\
\text { previous 12 months }\end{array}$ & 6 & 8 & 3 \\
$\quad$ Number of episodes of new & 0 & 0 & 0 \\
$\quad$ malarial cases & & & \\
Occupation & & & \\
$\quad$ Farming & 3 & 1 & 1 \\
$\quad$ Hotel & 1 & 2 & 2 \\
$\quad$ Student & 2 & 4 & 2 \\
$\quad$ Local leader & - & 1 & - \\
$\quad$ Teacher & - & 2 & - \\
\hline
\end{tabular}

Compliance with utilization. The devices distributed to family 1 ran out of batteries after 20 days. The family reached out to the GLI personnel, and we replaced the microgranule container and the batteries. The VPSs were never turned OFF during the day, as initially instructed. We repeated the education for each family. There were no further episodes of early depletion of granules during the evaluation.

Acceptability of the devices. Every individual was satisfied with the device performance. Families used the devices every night of the evaluation. They slept better because they did not have to swat mosquitos away at night. They did not report any difficulty in operating the VPS, and they were able to recharge the microgranule container and the batteries themselves after the second refill. They did not report any side effects such as coughing, wheezing, or skin reaction during the evaluation.

Device supply. We initially brought a sufficient number of devices and refills to conduct a 4-month evaluation. We then mailed refills for the remaining month from Europe. The devices were held by customs for 5 weeks because the device label described the products as an "insecticide diffuser." Uganda 2006 legislation requires every imported chemical to be registered, packed, and labeled in accordance with regulations stated by the law. ${ }^{9}$ The devices were eventually released after negotiations with the Uganda Ministry of Health.

Malaria. Twelve subjects (60\%) had at least one episode of malaria in the prior 12 months, data confirmed by a review of the Kagunga and Mugyera Health center records Three subjects developed symptoms compatible with malaria during the follow-up (fever, weakness, muscle and bone pain, nausea, and headaches). The RDT was negative, and the subjects were diagnosed with upper-respiratory tract infection. None of the subjects developed malaria during the initial 4-month follow-up. During the interval period of 5 weeks when we had experienced a delay in delivering the devices, three subjects were diagnosed with malaria after positive RDT and were treated according to the Uganda Ministry of Health guidelines. ${ }^{10}$ We did not observe further episodes of malaria during the fifth follow-up month.

In a survey of the records of the two clinics serving the villages, $20 \%$ of the population (61 of 604 individuals) was diagnosed and treated for malaria during the same 5-month study period. 
Reliability of the device. One device was found to be broken and nonfunctional at the end of the evaluation period. The remaining devices were operational. No device was disabled or stolen.

Costs. The cost of the VPS is approximately $\$ 14$, and the cost of each refill, which includes battery and micro-granules, is $\$ 9$. Given the expected duration of every single refill and the time we recommended to use the device, 12 hours a day, the cost per device would be $\$ 7$, per month.

The average annual cost of LLINs including delivery and retreatment ranges between $\$ 5.05$ and 10.08 , depending on the country and the program in charge of LLIN delivery. ${ }^{11}$ Considering that the average household size in rural Uganda is six units, according to the Ugandan Bureau of Statistics, ${ }^{12}$ the average cost of a LLIN per family varies between 30 and 60 dollars per year (\$2.5-5 per month).

\section{DISCUSSION}

The results of this exploratory intervention demonstrate that a battery-operated insecticide diffuser is a well-accepted device that could be used to prevent malaria in regions where malaria is endemic.

There is clinical and experimental evidence that spatial repellents can be effective in protecting against mosquitos. ${ }^{13}$ There are multiple commercially available devices including coils, vaporizer mats, ambient emanators, and liquid vaporizers. ${ }^{6}$ They have never been officially endorsed by the WHO because of environmental concerns. ${ }^{14,15}$ Their efficacy on preventing malaria is not proven, ${ }^{16,17}$ and their acceptance rate is also controversial. Most of these devices require an electrical outlet, a challenge in rural communities of subSaharan countries. The ownership and utilization of LLINs are most often well below the target set by governmental organizations. ${ }^{18}$ Individuals found it easy to use the VPS and did not have any problem replacing the batteries and the cartridges containing the micro-granules. The devices were reliable and delivered the insecticides when used accordingly to the instructions we provided.

An entomological survey carried out throughout Uganda in 2017-2019 showed that Anopheles gambiae was the principal vector in all but nine of the 71 health districts surveyed, and Plasmodium falciparum was detected in $5.5 \%$ of Anopheles. gambiae and in $4.0 \%$ of Anopheles. funestus examined. ${ }^{19}$ Knockdown resistance mutation Vgsc-L1014S was detected at a frequency of 0.96 in the sampled An. Gambiae. An. gambiae is also the dominant species of malaria vector in Kabale district. However, the documented resistance to pyrethroid in the Kabale region is only $14 \% .^{20}$ It is unclear whether the protection offered by the VAPE is because of its toxic or repellant effects. It is known that pyrethroids, in addition to a toxic effect, can repel by disorientation and prevent the host-seeking activity. ${ }^{21}$

We chose that specific area for this evaluation because, although located at an altitude of 1,950 m, malaria is present throughout the year because of the region's ecosystem. There is evidence that the climate changes that have been taking place over the last 30 years resulted in a temperature rise in some high-altitude regions of Africa, and consequently raised the elevation that experiences the threshold temperature required for malaria transmission. ${ }^{22,23}$ Also, we had access to support and coordination of a local non-governamental organization, the GLI, which has been an integral part of the local communities' lives for almost a decade. Their presence on the territory allowed us to conduct a much closer follow-up, repeated training, delivery of the devices, gathering data from the families and the nurses at the health centers, and negotiating with the local authority the release of the VPS.

The costs of the VPS are higher than those of currently used methodologies. This, however, does not take into account the costs of malaria-related deaths, Disability-Adjusted Life Year, and treatment costs. The costs we listed for the VPS are what consumers pay in a high-income country. The costs for bulk supply would probably be lower.

This report has several limitations. It was an exploratory evaluation of the acceptance and utilization of a new device to prevent malaria and not designed to evaluate its efficacy in preventing malaria. Although data seem to be promising, the very small sample and the lack of a proper control group do not allow drawing any conclusion on the efficacy of the device. A large-scale randomized trial is needed to assess the efficacy of this device in preventing malaria. This initial evaluation helped us to verify whether people would actually use the VPS and to identify difficulties to conduct future large-scale studies. They include the logistics of delivering the product in remote areas and the importance of repeated education to reinforce compliance with the specific intervention.

Received December 13, 2019. Accepted for publication January 24, 2020.

Published online February 24, 2020.

Authors' addresses: Giovanni Cucchiaro, Department of Anesthesiology and Critical Care Medicine, Johns Hopkins University, Johns Hopkins All Children's Hospital, St. Petersburg, FL, E-mail: gcucchi1@ jhmi.edu. Jamie Van Leeuwen, Global Livingston Institute, Denver, CO, E-mail: jamie@globallinvingstone.org. Yvette Goodridge, Department of Anesthesiology and Critical Care Medicine, Children's Hospital Los Angeles, Los Angeles, CA, E-mail: yvettegoodridge@ yahoo.com.

\section{REFERENCES}

1. Shretta R et al., 2017. Malaria elimination and eradication. Holmes KK, Bertozzi S, Bloom BR, Jha P, eds. Major Infectious Diseases. Washington, DC: World Bank.

2. World Health Organization, 2016. World Malaria Day $2016 »$ Key Facts. Washington, DC: National Institute of Allergy and Infectious Diseases. Available at: http://www.worldmalariaday. org/.

3. World Health Organization, 2017. World Malaria Report 2017. Geneva, Switzerland: WHO. Available at: http://apps.who.int/ iris/bitstream/handle/10665/259492/9789241565523-eng. pdf;jsessionid=D2DB40DB5FF7595CE02869F81E8E7C93? sequence $=1$.

4. Africa News, 2019. Uganda Records 1.4 Million Malaria Cases since June. Africa News. Available at: https://www.africanews.com/ 2019/08/15/uganda-records-14-million-malaria-cases-sincejune//.

5. Gonahasa S et al., 2018. LLIN evaluation in Uganda project (LLINEUP): factors associated with ownership and use of longlasting insecticidal nets in Uganda: a cross-sectional survey of 48 districts. Malar J 17: 421-436.

6. World Health Organization, 2006. Guidelines for Efficacy Testing of Household Insecticide Products. Geneva, Switzerland: WHO. Available at: http://www.who.int/whopes/resources/who_ htm_ntd_whopes_2009.3/en/.

7. Kegley SA, Hill BR, Orme S, Cho AH, 2010. PAN Pesticide Database. San Francisco, CA: Pesticide Action Network, North America. 
8. Van Leeuwen J, 2009. The Global Livingston Insitute. Available at: http://globallivingston.org/.

9. Parliament of Uganda, 2006. Agricultural Chemicals Control Act (2006). Available at: https://ulii.org/ug/legislation/act/2015/1-1.

10. Ministry of Health, 2016. Uganda Clinical Guidelines 2016. Available at: http://health.go.ug/sites/default/files/Uganda Clinical Guidelines 2016_FINAL.pdf.

11. Yukich $\mathrm{JO}$ et al., 2008. Costs and consequences of large-scale vector control for malaria. Malar J 7: 258-269.

12. Uganda Bureau of Statistics, 2014. Uganda National Household Survey. Kampala, Uganda: UBOS. Available at: http://www. ubos.org/onlinefiles/uploads/ubos/UNHS_12_13/2012_13 UNHS Final Report.pdf.

13. Ogoma SB, Moore SJ, Maia MF, 2012. A systematic review of mosquito coils and passive emanators: defining recommendations for spatial repellency testing methodologies. Parasit Vectors 5: 287-296.

14. Hogarh JN, Antwi-Agyei P, Obiri-Danso K, 2016. Application of mosquito repellent coils and associated self-reported health issues in Ghana. Malar J 15: 61-67.

15. John N, John J, 2015. Prolonged use of mosquito coil, mats, and liquidators: a review of its health implications. Int J Clin Exp Physiol 2: 209-213.

16. Avicor SW, Wajidi MFF, Owusu EO, 2017. To coil or not to coil: application practices, perception and efficacy of mosquito coils in a malaria-endemic community in Ghana. Environ Sci Pollut Res Int 24: 21138-21145.
17. Koenraadt CJ, Tuiten W, Sithiprasasna R, Kijchalao U, Jones JW, Scott TW, 2006. Dengue knowledge and practices and their impact on Aedes aegypti populations in Kamphaeng Phet, Thailand. Am J Trop Med Hyg 74: 692-700.

18. Aung T, Wei C, McFarland W, Aung YK, Khin HS, 2016. Ownership and use of insecticide-treated nets among people living in malaria endemic areas of eastern Myanmar. PLoS One 11: e0162292.

19. Lynd A et al., 2019. LLIN evaluation in Uganda project (LLINEUP): a cross-sectional survey of species diversity and insecticide resistance in 48 districts of Uganda. Parasit Vectors 12: 94-104.

20. USAID, 2016. President's Malaria Initiative, Uganda. Malaria Operational Plan FY 2016. Washington, DC: USAID. Available at: http://www.pmi.gov/docs/default-source/default-documentlibrary/malaria-operational-plans/fy16/fy-2016-uganda-malariaoperational-plan.pdf?sfvrsn $=5$.

21. Ritchie SA, Devine GJ, 2013. Confusion, knock-down and kill of Aedes aegypti using metofluthrin in domestic settings: a powerful tool to prevent dengue transmission? Parasit Vectors 6 : 262-271.

22. Lyon B, Dinku T, Raman A, Thomson MC, 2017. Temperature suitability for malaria climbing the Ethiopian Highlands. Environ Res Lett 12: 064015.

23. Siraj AS, Santos-Vega M, Bouma MJ, Yadeta D, Ruiz Carrascal D, Pascual M, 2014. Altitudinal changes in malaria incidence in highlands of Ethiopia and Colombia. Science 343: 1154-1158. 